\title{
Spin Fluctuations in the Stacked-Triangular Antiferromagnet $\mathrm{YMnO}_{3}{ }^{\text {II }}$
}

\author{
B. Roessli ${ }^{1}$, S. N. Gvasaliya ${ }^{1,2}$, E. Pomjakushina ${ }^{1,3}$, and K. Conder ${ }^{3}$ \\ ${ }^{1}$ Laboratory for Neutron Scattering ETHZ and Paul Scherrer Institute, CH-5232 Villigen PSI, Switzerland \\ ${ }^{2}$ Ioffe Physical Technical Institute, St. Petersburg, 194021 Russia \\ ${ }^{3}$ Laboratory for Development and Methods, Paul Scherrer Institute, CH-5232 Villigen PSI, Switzerland \\ Received February 24, 2005
}

\begin{abstract}
The spectrum of spin fluctuations in the stacked-triangular antiferromagnet $\mathrm{YMnO}_{3}$ was studied above the Néel temperature using both unpolarized and polarized inelastic neutron scattering. We find an in-plane and an outof-plane excitation. The in-plane mode has two components just above $T_{N}$ : a resolution-limited central peak and a Debye-like contribution. The quasi-elastic fluctuations have a line width that increases with $q$ as $D q^{z}$ and the dynamical exponent $z=2.3$. The out-of-plane fluctuations have a gap at the magnetic zone center and do not show any appreciable $q$ dependence at small wave vectors. (C) 2005 Pleiades Publishing, Inc.
\end{abstract}

PACS numbers: 75.40.-s, 78.70.Nx

\section{INTRODUCTION}

$\mathrm{YMnO}_{3}$ belongs to the family of $\mathrm{RMnO}_{3}(\mathrm{R}=$ Rareearth) manganite ferroelectric compounds that crystallize in the hexagonal space group $P 6_{3} \mathrm{~cm}$ below the paraelectric-ferroelectric phase transition ( 900 K). In $\mathrm{YMnO}_{3}$, the $\mathrm{Mn}^{3+}$ ions form triangular layers well separated from each other by $Y$ layers. Because, in the ferroelectric phase, the lattice is distorted, the Mn ions are slightly trimerized. The large separation between adjacent layers suggests that $\mathrm{YMnO}_{3}$ forms a good candidate for a geometrically frustrated 2-Dimensional (2D) antiferromagnet.

The magnetic structure of hexagonal $\mathrm{YMnO}_{3}$ was first investigated by Bertaut and Mercier [1] and reinvestigated later in more details by Munoz et al. [2]. Below $T_{N} \sim 70 \mathrm{~K}$, the $S=2$ magnetic moment of $\mathrm{Mn}$ ions are arranged in a $120^{\circ}$ magnetic structure with the triangular layers at $z=0$ and $z=1 / 2$ being antiferromagnetically coupled. At saturation, the magnetic moment is $\mu=2.9 \mu_{B}$; i.e., it is significantly reduced from the expected $4 \mu_{B}$ of $\mathrm{Mn}^{3+}$ spins, which was taken as evidence that, even in the ordered phase, strong spin fluctuations are present as a consequence of geometrical frustration [3]. Analysis of the spin-wave spectrum has confirmed the 2D character of the magnetic exchange interactions in $\mathrm{YMnO}_{3}$ [4] with the ratio of the intra- to interplane exchange interactions being on the order $\sim 2 \times 10^{2}$. Whereas well-defined excitations are observed below the ordering temperature, a broad inelastic signal, as well as short-range correlations between the Mn magnetic moments within the triangular layers, persists well above $T_{N}$ [3].

\footnotetext{
II This article was submitted by the authors in English.
}

In this work, we investigate the $q$ and temperature dependence of the spin excitations in $\mathrm{YMnO}_{3}$ close to the Néel temperature. We were motivated by the fact that the nature of the phase transition of frustrated magnets is still not completely understood and that the critical properties of stacked triangular antiferromagnets have received special attention since Kawamura [5] proposed that the critical exponents in these systems form a new universality class. Experimental confirmation of the new class of (chiral) exponents was found in $\mathrm{CsMnBr}_{3}$ by unpolarized [6] and polarized neutron scattering [7] measurements as well as in Ho [8]. Second, an anomaly in the dielectric constant $\varepsilon$ with the electric field applied in the $a b$ plane was found in $\mathrm{YMnO}_{3}$ [9] at $T_{N}$. The nature of the coupling between the electric and magnetic properties in hexagonal manganites is a subject of intense debate [10]. Clearly, it is required to characterise the behavior of the magnetic fluctuations in the vicinity of $T_{N}$ to understand the possible relationship with the magneto-dielectric effect in $\mathrm{YMnO}_{3}[11]$.

\section{EXPERIMENTAL}

Polycrystalline $\mathrm{YMnO}_{3}$ was prepared using a solid state reaction. The starting materials of $\mathrm{Y}_{2} \mathrm{O}_{3}$ and $\mathrm{MnO}_{2}$ with $99.99 \%$ purity were mixed and grounded and then treated at $1000-1200^{\circ} \mathrm{C}$ in air for at least $70 \mathrm{~h}$ with several intermediate grindings. The phase purity of the compound was checked with a conventional $x$-ray diffractometer (SIEMENS D500). The powder was hydrostatically pressed in the form of rods $(8 \mathrm{~mm}$ in diameter and $\sim 60 \mathrm{~mm}$ in length). The rods were subsequently sintered at $1300^{\circ} \mathrm{C}$ for $30 \mathrm{~h}$. The crystal growth was carried out using an optical floating zone furnace (FZ-T- 


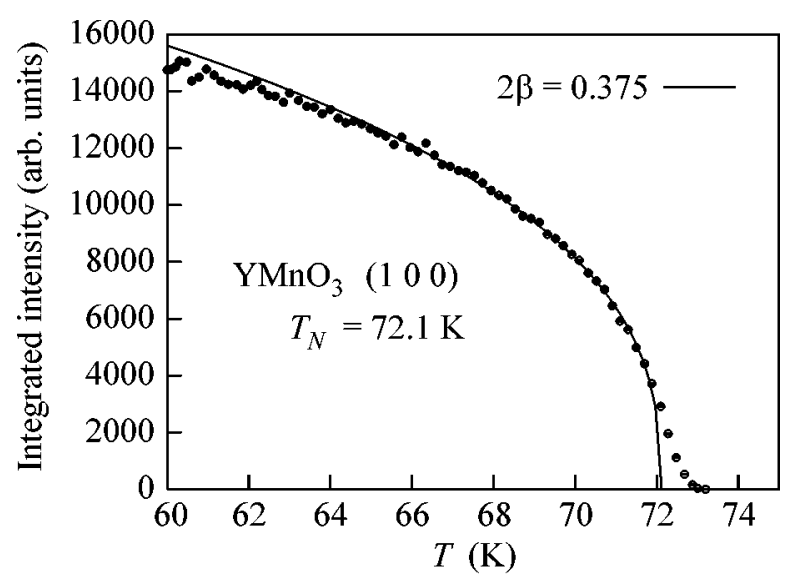

Fig. 1. Temperature dependence of the intensity of the $\left(\begin{array}{lll}1 & 0 & 0\end{array}\right)$ magnetic Bragg peak. The line is a fit to the data with a power law.

10000-H-IV-VP-PC, Crystal System Corp., Japan) with four $1000 \mathrm{~W}$ halogen lamps as a heat source. The growth rate was $1.5 \mathrm{~mm} / \mathrm{h}$ and both rods (the feeding and seeding rod) were rotated at about $20 \mathrm{rpm}$ in opposite directions to ensure the liquid homogeneity. A mixture of argon with $2 \%$ oxygen at 5.5 bar was applied during the growing. The crystal has the shape of a rod $6 \mathrm{~mm}$ in diameter and $2 \mathrm{~cm}$ in height and a mosaic spread better than $1^{\circ}$.

The measurements were performed at triple-axis TASP located on the cold source of the neutron spallation source SINQ. The sample was mounted inside an He-flow cryostat with the crystallographic axis $a^{*}$ and $c^{*}$ in the scattering plane. The spectrometer was operated with the energy of the scattered neutrons kept fixed at $k_{f}=1.47 \AA^{-1}$ for measurements with unpolarized neutrons. 80' Soller collimators were installed in the incident beam and before the analyzer and the detector. With that configuration the energy resolution at zero energy transfer is $180 \mu \mathrm{eV}$. To reduce both the background and contamination by higher wavelength neutrons a cold Be filter was installed in the scattered beam. The inelastic polarized neutron measurements were performed at $k_{f}=1.51 \AA^{-1}$ along $(1 \pm q, 0,0.1)$ and at different temperatures above $T_{N}$. To perform longitudinal-polarization analysis, remanent supermirror benders [12] were inserted after the monochromator and before the analyzer. The orientation of the polarization was chosen perpendicular to the scattering plane. Because magnetic fluctuations with a polarization factor parallel to the neutron spin occur in the nonspin flip channel, the nonspin flip data (NSF) contains the inplane fluctuations, and, in the spin-flip channel (SF), only out-of-plane fluctuations are present, as will be shown below.

\section{RESULTS AND DISCUSSION}

Figure 1 shows the intensity of the ( $\left.\begin{array}{lll}1 & 0 & 0\end{array}\right)$ magnetic Bragg reflection as a function of the temperature, which mirrors the square of the staggered magnetization. The transition temperature, as determined by taking the derivative of the magnetization curve [13], is $T_{N}=$ $72.1 \pm 0.05 \mathrm{~K}$. The intensity of the Bragg peak follows the power law $I \propto\left|\left(T / T_{N}-1\right)\right|^{2 \beta}$ with $\beta=0.187(2)$. This value is lower than that reported for the case of typical stacked-triangular antiferromagnet $\operatorname{RbNiCl}_{3}(\beta=0.28)$, $\mathrm{CsNiCl}_{3}(\beta=0.28)$, and $\mathrm{CsMnBr}_{3}(0.21<\beta<0.25)$ and close to the critical exponent obtained in $\mathrm{VCl}_{2}(\beta=$ 0.20) $[5,14]$. Typical 2D $X Y$-antiferromagnets $\left(\mathrm{BaNi}_{2}\left(\mathrm{PO}_{4}\right)_{2}\right)$ or ferromagnets $\left(\mathrm{Rb}_{2} \mathrm{CrCl}_{4}, \mathrm{~K}_{2} \mathrm{CuF}_{4}\right)$ have a magnetization exponent that corresponds to the expected theoretical value $\beta=0.23$ [15]. We note that $\beta=0.19$ in the $3 \mathrm{D}$ triangular Ising antiferromagnet [16].

Figure 2a shows elastic scans along the $\left[\begin{array}{lll}1 & 0 l\end{array}\right]$ direction as a function of temperature. Close to the Néel temperature, correlations between adjacent hexagonal planes give rise to broad magnetic scattering along $c^{*}$ that eventually appears to condense into the magnetic Bragg peak at $T_{N}$. From Fig. $2 \mathrm{~b}$, we see that this diffuse scattering is static on the time scale of our experiment and corresponds to slow fluctuations of in-plane character, as will be shown below. With increasing temperature, these correlations disappear and only scattering across the $\left[\begin{array}{lll}1 & 0 & l\end{array}\right]$ direction show a peak in the neutron cross section. Hence, well above $T_{N}$, correlations persist only between the Mn moments located in the hexagonal plane, as shown in Fig. 3. Here, we describe the line shape of the diffuse intensity by a Lorentzian profile convoluted with the resolution function of the spectrometer:

$$
\chi_{c}(\mathbf{Q})=\frac{\chi_{0}}{\pi} \frac{\kappa_{\|}^{2}}{\left(\mathbf{q}_{\|}-\mathbf{Q}_{0}\right)+\kappa_{\|}^{2}} \frac{\kappa_{\perp}^{2}}{\left(\mathbf{q}_{\perp}-\mathbf{Q}_{0}\right)^{2}+\kappa_{\perp}^{2}} \delta(\omega),
$$

where $\mathbf{Q}_{0}$ is the position of the magnetic rod in reciprocal space, $\|$ and $\perp$ denote the direction along and perpendicular to the magnetic rod, and $\kappa_{\|}$denotes the inverse of the correlation length $\xi$ between Mn spins in the hexagonal plane. Close to the Néel temperature, the temperature dependence of $\xi$ behaves similar to $\xi(T)=$ $0.038( \pm 0.005)\left(T-T_{N}\right)^{v}$ with $v=0.57( \pm 0.06)$ as shown in Fig. 3b. We now turn to the behavior of the paramagnetic fluctuations in $\mathrm{YMnO}_{3}$. Figure 4 shows typical energy scans performed at $\mathbf{Q}=(1-q, 0,-3.8)$ and $T=$ $73.6 \mathrm{~K}$. The inelastic cross-section for an unpolarized neutron beam is given by

$$
\frac{d^{2} \sigma}{d \Omega d \omega} \propto \sum_{\alpha \beta}\left(\delta_{\alpha \beta}-\hat{Q}_{\alpha} \hat{Q}_{\beta}\right) S^{\alpha \beta}(\mathbf{Q}, \omega),
$$

where $\delta_{\alpha \beta}$ is the Kronecker symbol; $\alpha, \beta$ are the Cartesian coordinates $x, y, z,(\mathbf{Q}, \omega)$ that denote the momen- 


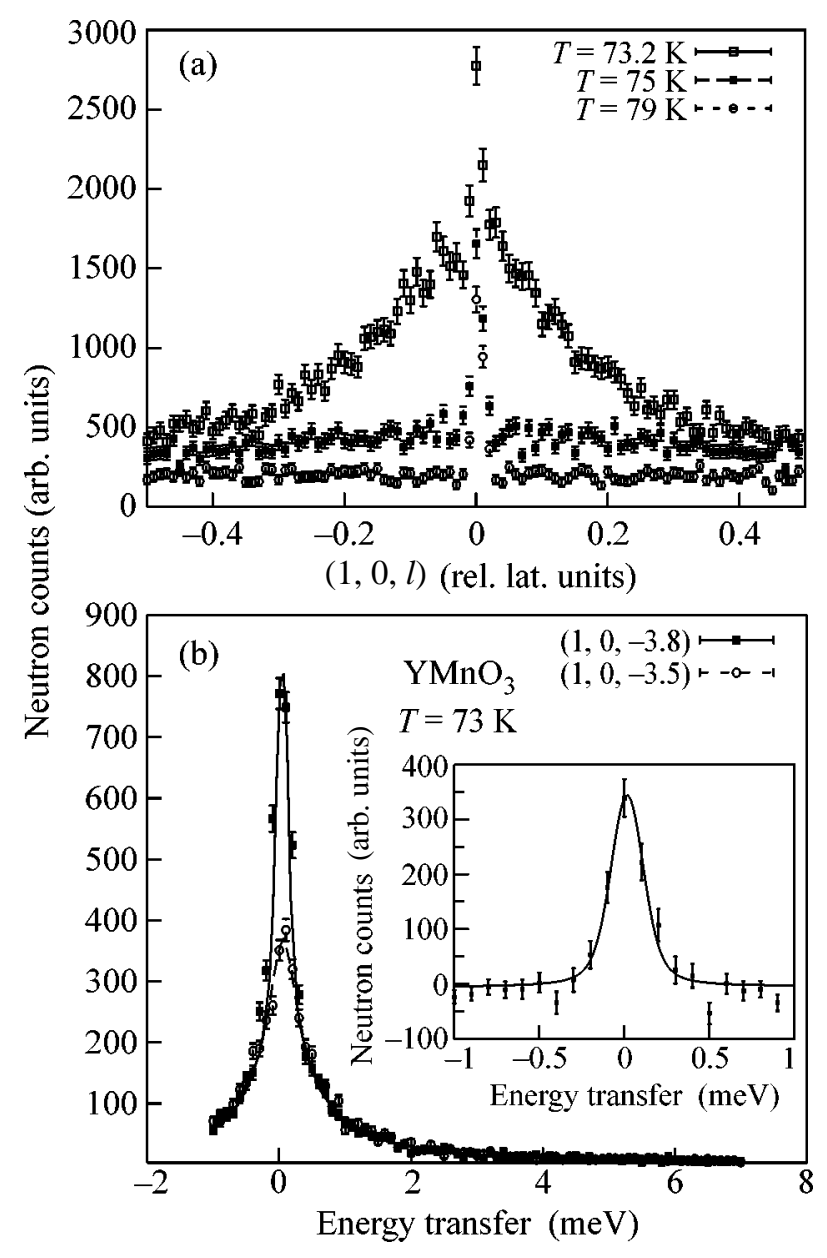

Fig. 2. (a) Elastic scans along $(1,0, l)$ that show the increase of diffuse magnetic scattering in $\mathrm{YMnO}_{3}$ when the temperature approaches $T_{N}$. (b) Inelastic scan that shows the presence of the central peak in $\mathrm{YMnO}_{3}$. The insert shows the difference between scans measured at $(1,0,-3.8)$ and $(1,0$, $-3.5)$ that emphasizes the resolution-limited component.

tum and energy transfers from the neutron to the sample; and $\hat{Q}=\mathbf{Q} /|\mathbf{Q}|$. The first term in Eq. (2) is a selection rule that implies that only spin components perpendicular to the scattering vector contribute to the neutron scattering cross section. Hence, for scattering vectors with large $l$ components such as $\mathbf{Q}=(1 \pm q, 0$, $-3.8)$, the inelastic spectrum contains essentially paramagnetic fluctuations with in-plane character $(\|)$. In addition, close to the Néel temperature, inelastic scans through the magnetic rod show the resolution-limited central peak described by Eq. (1) as shown in Fig. 2. Thus, to analyze the data shown in Fig. 4, we modeled the inelastic intensity $I(\mathbf{Q}, \omega)$ in the following way:

$$
\begin{gathered}
I(\mathbf{Q}, \omega)=\left(S_{\text {para }}^{\|}(\mathbf{Q}, \omega)\right. \\
\left.+S_{\text {inc }}(\omega)+S \chi_{c}(\mathbf{Q})\right) \otimes R(\mathbf{Q}, \omega)+B c k,
\end{gathered}
$$

where $S_{\text {inc }}=A \delta(\omega)$ refers to the resolution-limited incoherent scattering that was measured at high temperature
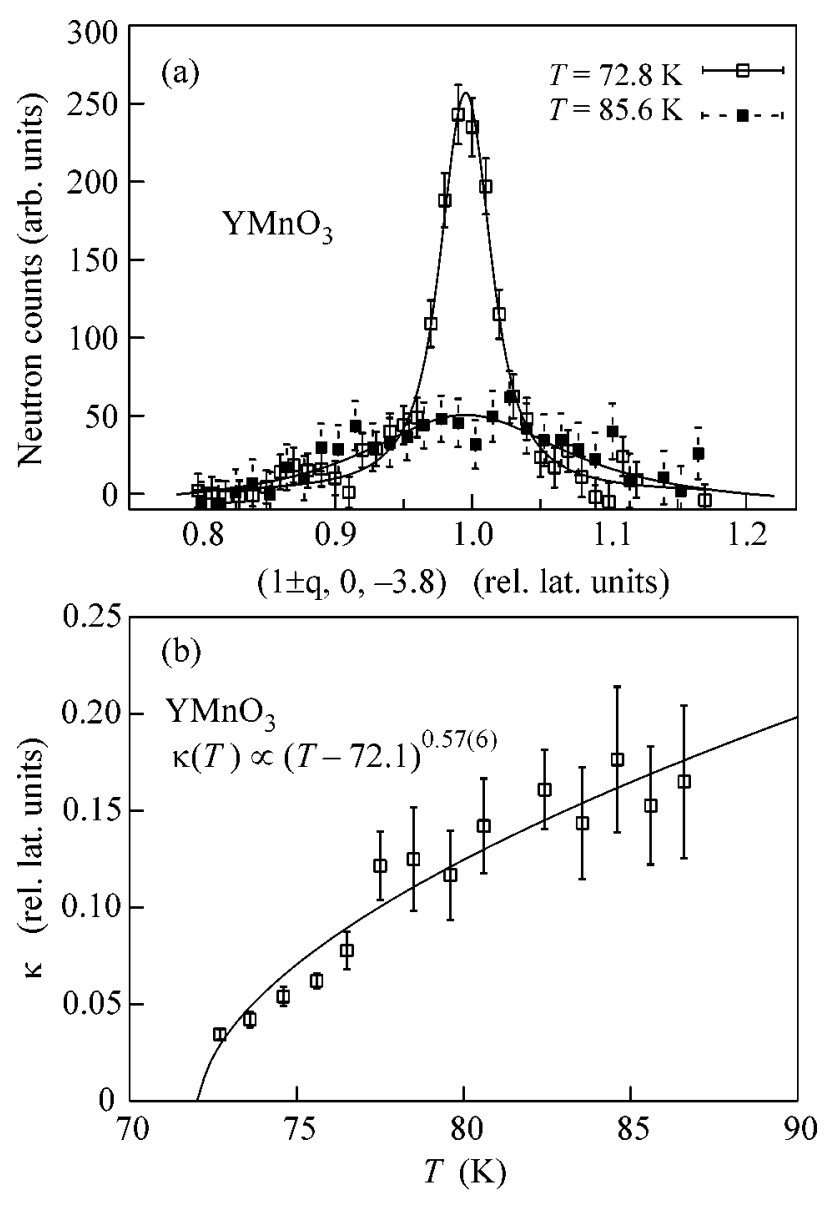

Fig. 3. (a) Neutron diffuse intensity in $\mathrm{YMnO}_{3}$. The background was measured at $T=300 \mathrm{~K}$ and has been subtracted. (b) Temperature dependence of the inverse of the correlation length. The line is a fit to the data with a power law.

and $S$ is a scale factor. The neutron scattering function $S_{\text {para }}^{\|}(\mathbf{Q}, \omega)$, which is related to the imaginary part of the dynamical susceptibility through $\pi\left(g \mu_{B}\right)^{2} S_{\text {para }}^{\|}(\mathbf{Q}, \omega)=$ $F^{2}(Q)\left(1-\exp \left(-\hbar \omega / k_{B} T\right)\right)^{-1} \Im \chi^{\prime \prime}(\mathbf{Q}, \omega)$, describes the line shape of the paramagnetic scattering as a function of momentum $(\mathbf{Q})$ and energy $(\hbar \omega)$ transfer, respectively; $F(Q)$ is the magnetic form factor of Mn. In Eq. (3), the symbol $\otimes$ stands for the convolution with the spectrometer resolution function $R(\mathbf{Q}, \omega)$ [17] and $B c k$ denotes the background level. We find that a Debye-like quasi-elastic line shape for the imaginary part of the dynamical susceptibility

$$
\Im \chi^{\prime \prime}\left(\mathbf{Q}_{0}+\mathbf{q}, \omega\right)=\omega \chi\left(\mathbf{Q}_{0}+\mathbf{q}\right) \frac{\Gamma(\mathbf{q})}{\omega^{2}+\Gamma(\mathbf{q})^{2}}
$$

reproduces the data adequately. $\chi(\mathbf{q})$ is the static susceptibility as in Eq. (1) taken relative to the antiferromagnetic zone center $\left(\mathbf{Q}_{0}=(1,0,0)\right)$, and $\Gamma(\mathbf{q})$ is the damping of the paramagnetic fluctuations. Figure 4 


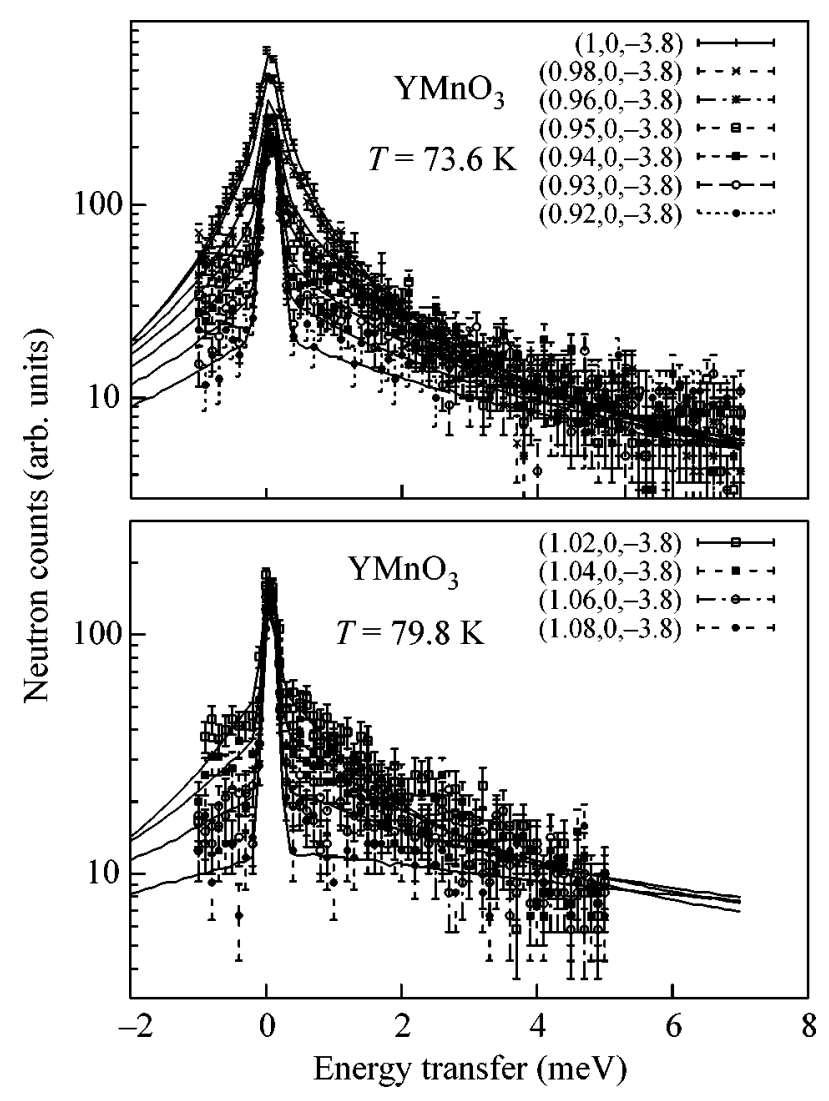

Fig. 4. Constant $q$-scans in $\mathrm{YMnO}_{3}$ at $T=73.6 \mathrm{~K}$ and $79.8 \mathrm{~K}$ that show the in-plane fluctuations, as explained in the text. The lines are the result of fit to the data using Eq. (3).

shows the results of fits to the data at $T=73.6 \mathrm{~K}$ from which we extract that the damping of the in-plane fluctuations $\Gamma(\mathbf{q})$ evolves similar to $\Gamma(0, T)+(1450 \pm 90) q^{z}$ $(\mathrm{meV})$ with $\Gamma(0,73.6 \mathrm{~K})=0.23 \pm 0.07(\mathrm{meV})$, and $z=$ $2.26 \pm 0.07$. In contrast with data taken around $(1 \pm q$, $0,3.8)$, the inelastic spectra around $(1 \pm q, 0,0.1)$ cannot be fitted with Eq. (4). This is an indication that the spectrum of paramagnetic fluctuations in $\mathrm{YMnO}_{3}$ consists of 2 modes, an in-plane $(\|)$ as well as an out-ofplane component $(\perp)$. To separate the $\|$ from the $\perp$ fluctuations, it is necessary to use polarization analysis. A typical inelastic spectrum measured with polarization analysis is shown in Fig. 5 that reveals an out-of-plane excitation in the spin-flip channel. In contrast with the

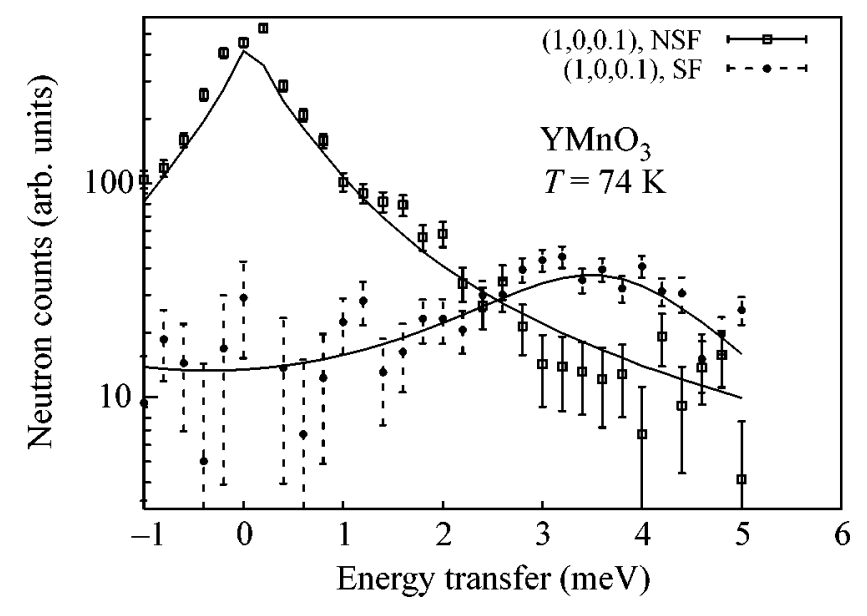

Fig. 5. Constant $q$-scans in $\mathrm{YMnO}_{3}$ at $T=73.6 \mathrm{~K}$ that show the in-plane fluctuations in the non-spin flip (NSF) and the out-of-plane component in the spin-flip channel (SF), respectively. The lines are the result of fit to the data as described in the text.

\|l fluctuations, the $\perp$ component is inelastic and is best described by a damped-harmonic oscillator function

$$
S_{D H O}^{\perp} \propto \frac{\omega}{1-\exp (-\omega / T)} \frac{\gamma_{q}}{\left(\omega^{2}-\Omega_{q}^{2}\right)^{2}+\omega^{2} \gamma_{q}^{2}},
$$

where $\Omega_{q}$ is the renormalized frequency and $\gamma_{q}$ the damping of the excitation. Within the precision of the measurements, we find that $\Omega_{q}=3.9 \pm 0.3(\mathrm{meV})$ and $\gamma_{q}=2 \pm 0.5(\mathrm{meV})$ in the range of momentum values reached in the present experiment $(q \leq 0.1)$.

The analysis of the data in the non-spin-flip channel yields the same $q$ dependence for the $\|$ fluctuations that was obtained with the unpolarized set up. The results are summarized in the table. The mean value for the dynamical exponent of the $\|$ fluctuations in $\mathrm{YMnO}_{3}$ yields $z \sim 2.3$ and does not agree with the theoretical dynamical exponent $z=1$ for the classical 2D triangular antiferromagnet [18]. Also, the value obtained in $\mathrm{YMnO}_{3}$ is quite different from the dynamical exponents $z=1.5$ expected for the 3D Heisenberg antiferromagnet and measured, e.g., in $\mathrm{RbMnF}_{3}$ [19] and $\mathrm{CsMnBr}_{3}$ [20]. Finally, we show the temperature dependence of $\Gamma(0, T)$ in Fig. 6, which increases almost linearly above $T_{N}, \Gamma(0, T)=0.4+0.07\left(T-T_{N}\right)^{1.1 \pm 0.2}$.

Parameter values for the damping $\left(\Gamma(\mathbf{q})=\gamma q^{z}(\mathrm{meV})\right)$ and the critical exponent $(z)$ of the $\|$-fluctuation in $\mathrm{YMnO}_{3}$

\begin{tabular}{l|l|l|l|l|l}
\hline & $T(\mathrm{~K})$ & $\gamma$ & $\Delta \gamma$ & $z$ & $\Delta z$ \\
\hline Unpol. & 73.6 & 1450 & 90 & 2.26 & 0.07 \\
& 79.8 & 1283 & 272 & 2.29 & 0.16 \\
Pol. & 74 & 1306 & 277 & 2.53 & 0.25 \\
& 78 & 1028 & 313 & 2.40 & 0.4 \\
\hline
\end{tabular}




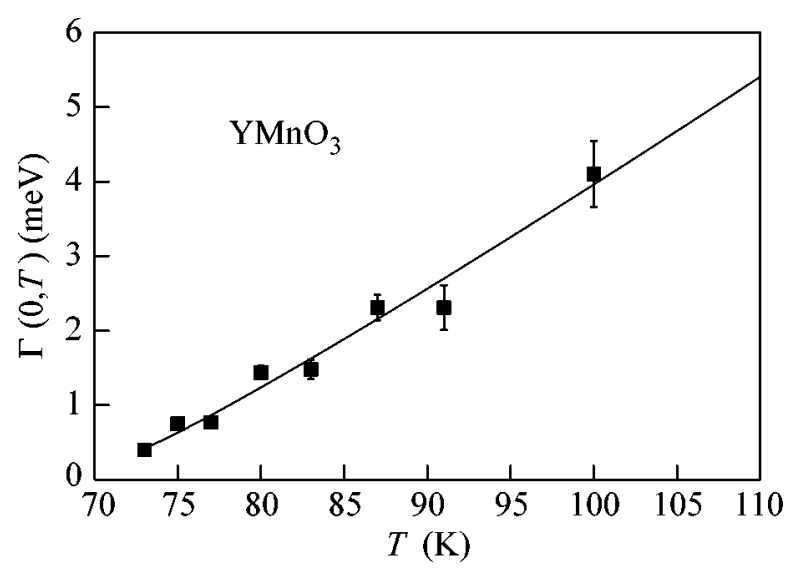

Fig. 6. Temperature dependence of the damping of the paramagnetic fluctuations with in-plane polarization as measured with polarized neutrons. The line is a fit to the data as explained in the text.

\section{CONCLUSIONS}

To conclude, using both unpolarized and polarized inelastic neutron scattering, we showed that there are two magnetic excitations in the paramagnetic regime of $\mathrm{YMnO}_{3}$ that have in-plane and out-of-plane polarization, respectively. The in-plane mode has a resolutionlimited central peak and a quasi-elastic component with a line width that increases as a function of momentum transfer $q$ similar to $\propto q^{z}$ with the dynamical exponent $z=2.3$. The presence of two time scales in the spectrum of in-plane fluctuations might be the signature for the coexistence of 2D and 3D fluctuations in the vicinity of $T_{N}$ [21]. The out-of-plane fluctuations are inelastic at the magnetic zone center and do not show any $q$ dependence for small wave vectors.

This work was performed at the Neutron Spallation Source SINQ, Paul Scherrer Institut, Switzerland and was partly supported by the NCCR MaNEP project.

\section{REFERENCES}

1. B. Bertaut and M. Mercier, Phys. Lett. 5, 27 (1963).
2. A. Munoz, J. A. Alonso, M. J. Martínez-Lope, et al., Phys. Rev. B 62, 9498 (2000).

3. Junghwan Park, J.-G. Park, Gun Sang Jeon, et al., Phys. Rev. B 68, 104426 (2003).

4. T. J. Sato, S. H. Lee, T. Katsufuji, et al., Phys. Rev. B 68 , 014432 (2003).

5. For a recent review see H. Kawamura, J. Phys.: Condens. Matter 10, 4707 (1998).

6. T. E. Mason, B. D. Gaulin, and M. F. Collins, Phys. Rev. B 39, 586 (1989).

7. V. P. Plakhty et al., Europhys. Lett. 48, 215 (1999); Phys. Rev. Lett. 85, 3942 (2000).

8. V. P. Plakhty, W. Schweika, Th. Brückel, et al., Phys. Rev. B 64, 100402 (2001).

9. Z. J. Huang et al., Phys. Rev. B 56, 2623 (1997); T. Katsufuji et al., Phys. Rev. B 64, 104419 (2001).

10. T. Kimura et al., Nature 426, 55 (2003); D. V. Efremov et al., Nat. Mater. 3, 853 (2004); B. Lorenz et al., Phys. Rev. Lett. 92, 087204 (2004).

11. G. Laws, A. P. Ramirez, C. M. Varma, et al., Phys. Rev. Lett. 91, 257208 (2003).

12. F. Semadeni, B. Roessli, and P. Böni, Physica B (Amsterdam) 297, 152 (2001).

13. A. D. Bruce, J. Phys. C 14, 193 (1982).

14. M. F. Collins and O. A. Petranko, Can. J. Phys. 75, 605 (1997).

15. S. T. Bramwell and P. C. W. Holdsworth, J. Phys.: Condens. Matter 5, L53 (1993).

16. O. Heinonen and R. G. Petschek, Phys. Rev. B 40, 9052 (1989).

17. M. Popovichi, Acta Crystallogr. A 31, 507 (1975).

18. Kwangsik Nho and D. P. Landau, Phys. Rev. B 66, 174403 (2002).

19. R. Coldea, R. A. Cowley, T. G. Perring, et al., Phys. Rev. B 57, 5281 (1998).

20. T. E. Mason, Y. S. Yang, M. F. Collins, et al., J. Magn. Magn. Mater. 104-107, 197 (1992).

21. L. P. Regnault, C. Lartigue, J. F. Legrand, et al., Physica B (Amsterdam) 156-157, 298 (1989); A. R. Völkel, A. R. Bishop, F. G. Mertens, et al., J. Phys.: Condens. Matter 4, 9411 (1992). 